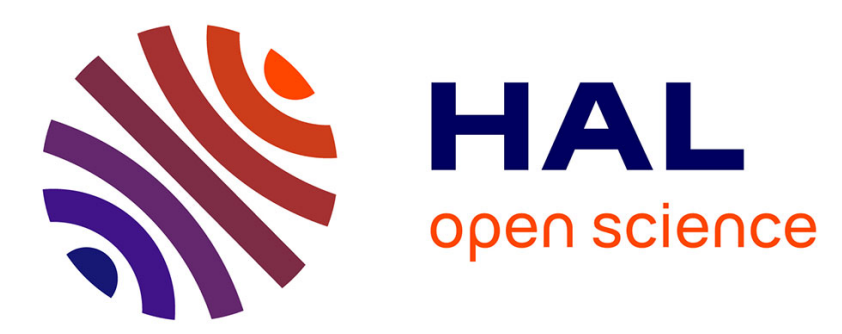

\title{
Initialized Iterative Closest Point for bone recognition in ultrasound volumes
}

\author{
Oussama Haddad, Julien Leboucher, Jocelyne Troccaz, Eric Stindel
}

\section{To cite this version:}

Oussama Haddad, Julien Leboucher, Jocelyne Troccaz, Eric Stindel. Initialized Iterative Closest Point for bone recognition in ultrasound volumes. International Conference on Pattern Recognition, Dec 2016, Cancun, Mexico. hal-01455683

\section{HAL Id: hal-01455683 \\ https://hal.univ-grenoble-alpes.fr/hal-01455683}

Submitted on 3 Feb 2017

HAL is a multi-disciplinary open access archive for the deposit and dissemination of scientific research documents, whether they are published or not. The documents may come from teaching and research institutions in France or abroad, or from public or private research centers.
L'archive ouverte pluridisciplinaire HAL, est destinée au dépôt et à la diffusion de documents scientifiques de niveau recherche, publiés ou non, émanant des établissements d'enseignement et de recherche français ou étrangers, des laboratoires publics ou privés. 


\title{
Initialized Iterative Closest Point for bone recognition in ultrasound volumes
}

\author{
Oussama Haddad $^{1,2}$, Julien Leboucher ${ }^{1}$, Jocelyne Troccaz ${ }^{2}$ and Eric Stindel ${ }^{1,3}$ \\ ${ }^{1}$ Laboratoire de Traitement de l'Information Médicale LATIM INSERM, Brest, France. \\ ${ }^{2}$ Univ.Grenoble Alpes/ CNRS / TIMC-IMAG UMR 5525, Grenoble, F-38041, France. \\ ${ }^{3}$ Centre Hospitalier Régional et Universitaire, Service Orthopédie Traumatologie, Brest, France.
}

\begin{abstract}
Ultrasound (US) probes have been used as guiding tools for Computer Assisted Orthopedic Surgeries (CAOS) [1]. Because of the US data uncertainty, the process of recognition - the localization of regions of interest in the image- requires a registration to a more precise, but invasive, imaging modality such as Computed Tomography $(C T)$. A millimetric precision and a real-time processing are intraoperative requirements. Iterative Closest Point (ICP) [2] is a simple and non symmetric rigid registration algorithm that is sensitive to the initial position of the point sets. The aim of this study is to show the contribution of initializing ICP in rigid US-CT registration and to illustrate it on data of a proximal femur. First, an iterative initialization of the model (CT) to the partial view (US) is performed using ICP with annealed filtering. The first obtained local minimum is then used to initialize a refinement step that maps the partial view to the model. One femur phantom was imaged both in a water bath using a calibrated $3 D$ ultrasound probe and by CT. For each of the ten US acquisitions (five in the Anterior neck $A$, and five in the Posterior neck P), the CT scan is brought by means of fiducials pair-point matching. The initialization step improves ICP successful registrations from (A:25\%, P:21\%) to (A:76\%, to P:52\%) and the registration takes about $3 \mathrm{~s}$ in average whilst ICP takes about $1 \mathrm{~s}$.
\end{abstract}

\section{Introduction}

Skin markers [3], time of flight cameras [4] and ultrasound probes [5] have been proposed as guiding tools in non invasive CAOS. US based guidance for CAOS generally relies on a prior knowledge on the bone shape that is elaborated in a preoperative step of the surgery. In the Operating Room (OR), a fast and accurate transfer of a preoperative plan helps the surgeon in locating regions of interests in the bone.

ICP is a simple and fast rigid registration method. It is based on the iterative search of point to point correspondences in sets of 3D points. It is used to map a partial view (source) to a model (destination). Non suitable initial state and incorrect correspondences are major sources of ICP failure. Points in the model that have no homologues are ignored in the iterative registration process. The full model information can be used in the initialization. Obtaining a suitable initial state for ICP can be done by matching anatomical landmarks [6]. Landmarks can be extracted manually, which hinders the "real-time" constraint. Curvilinear features (such as the curvature) of the meshes [7], challenging to extract from US data, have also been proposed. Matching principal axes of the meshes was also used $[8,9]$, but non overlapping regions in CT scans and US noise change the estimate of the principal axes. Genetic algorithms are a possible way to initialize ICP [10] but complexity is a critical issue. Recently [11], nested annealing was used to initialize ICP. High convergence rates and running times were reported. It was also reported that the proposed method experiences troubles aligning symmetric shapes, since local minima would resemble to the global one. Generally, the annealing process can be used as a tool to help iterative algorithms traverse local minima. In previous work [8], we showed that it helps ICP in the non-overlapping regions detection.

Registration performances are also affected by ultrasound noise. Preprocessing steps were proposed in order to better reconstruct US data: Poisson [8], anisotropic diffusion [12] and weighting the reconstruction using the cosine of the incidence angle [13].

In this paper, an initialized ICP approach is tested for USCT registration of proximal femur. An iterative initialization maps the model to the partial view using ICP. The minimized cost function is made convex by the use of US point's confidence in an annealing process. The first obtained local minimum is then used to initialize a refinement step that maps the partial view to the model. Data are collected using a CT scanner and a localized 3D ultrasound probe imaging a femur phantom fixed in a water bath. Registration results are evaluated using a ground truth given by fiducial pair-point matching.

\section{Registration in ultrasound based CAOS}

Non invasive means to track bones include: skin markers, Time of Flight or RGB-D cameras, ultrasound probes. This study focuses on 3D US. US to CT image registration aims at transferring pre-operative information and planning in the OR. The Fig 1 shows major steps in US-based CAOS. 


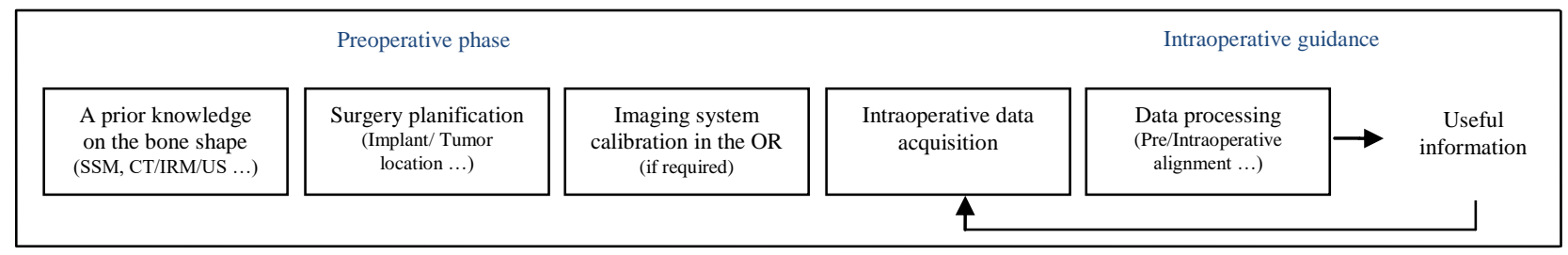

Fig 1: Pre-Intraoperative steps of an ultrasound based CAOS

\subsection{Preoperative steps}

Medical decisions prior to the surgery and a prior knowledge on the bone shape need to be available to the guidance system in the OR. Statistical Shape Models (SSM) or CT/MRI acquisitions are used for this purpose. The intraoperative imaging tool is a tracked 3D ultrasound probe. A probe to tracker calibration is then required [14].

\subsection{Intraoperative guidance}

The matching between intraoperative US and preoperative data helps localizing a target visible on the preoperative data in the OR. Acquiring and processing intraoperative data is constrained by accuracy (millimetric to be able to replace existing invasive fiducials [15]) and speed (processing must not take longer than the acquisition). The process has to be repeatable within the surgery time frame if required.

\section{Methods}

\subsection{ICP}

Consider the problem of positioning a shape $\mathrm{X}$ in a model $\mathrm{Y}$ (represented by $\mathrm{n}$ and $\mathrm{m}$ points respectively). ICP iteratively provides an estimate of the optimal transformation $\mathrm{T}$ that maps $\mathrm{X}$ (source) to $\mathrm{Y}$ (destination). It minimizes a Surface Registration Error (SRE):

$$
T=\operatorname{argmin}_{t}\left(S R E=\frac{1}{n} \sum_{i=1}^{n}\left\|y_{i}-t\left(x_{i}\right)\right\|\right)(1)
$$

Where $y_{i}$ is the nearest point in set $\mathrm{Y}$ to $t\left(x_{i}\right)$. Equation (1) yields to a lack of robustness to noise which is mainly ultrasound noise in this study. A commonly used strategy is to introduce a weighting parameter related to the quality of the match $[8,16]$. We use the cosine of the incidence angle $\left(\alpha_{i}\right)$ [15] between the line joining the US emitter to the surface point and the local surface normal at this point.

$$
\begin{gathered}
T=\operatorname{argmin}_{t}\left(S R E=\frac{1}{n} \sum_{i=1}^{n} w_{i}\left\|y_{i}-t\left(x_{i}\right)\right\|\right) \\
w_{i}=\left|\cos \alpha_{i}\right|(3)
\end{gathered}
$$

A visualization of the obtained reference positions is provided in Fig 4. A color map shows the value of (3) at each
US point. Important factors influence the registration accuracy: The surface areas selected for registration (which can be controlled by the FoV of the US emitter), the quality of the meshes (which can be improved by elaborating a more sophisticated segmentation, for example local phase features for US bones detection [17]), and the initial estimate of the correspondences.

\subsection{Iterative initialization}

In ICP, using the model as the source shape can provide more information to the registration process, where all the model points participate in the estimate of the optimal transform. In this case, the minimized SRE includes nonoverlapping regions, and convergence is not guaranteed. We propose to use the model as the source shape in an iterative initialization. The registration is guided by the confidences accorded to the US points. Initially, all correspondences are given the same weight 1 . Iteratively, the filtering effect increases using deterministic annealing and following:

$$
w_{i}=\left|\cos \alpha_{i}\right|^{1-r^{\text {Number }} \text { of iteration }}
$$

Where $r$ is the annealing rate, a compromise between accuracy and runtime. We fix $r=0.93$ similarly to [9]. Mapping CT points to US correspondences using ordered weights is done using Singular Value Decomposition [18]. The first obtained local minimum (SRE starts increasing or stabilizing) is used to initialize the refinement step: The obtained optimal transformation that maps the CT to US, is inverted and applied on the US mesh (Initialized US). The latter is registered to the original CT using standard ICP. This way a comparison to classic ICP becomes undemanding.

\section{Data \\ 4.1. CT Data}

A CT scan of a femur phantom (sawbones ${ }^{\circledR}$ ) (Siemens Biograph, with an imaging resolution of $0.7 \mathrm{~mm}$ ) is used as the model in the study. Thirteen 1-mm metal fiducials were attached to the bone. Thresholding segmentation was performed and a 3D surface mesh was extracted from the segmented CT volume. The mesh was then filtered using Laplace smoothing [19]. We only keep the proximal part of the femur ( $\frac{1}{6}$ over the surface of the entire bone, $100 \mathrm{~cm}^{2}$ 
calculated as the sum of the triangles surfaces, and 29.000 points).

\subsection{Ultrasound data}

Ultrasound data are obtained using a tracked 3D transducer (Voluson 730 ultrasound machine, GE Medical Systems) imaging the femur phantom fixed in a water tank. The Field of View (FoV) is $90^{\circ}$, the depth is $7 \mathrm{~cm}$ and the resolution is $0.47 \mathrm{~mm}$. Probe calibration [14] was performed using a membrane phantom in nylon. It enables to define a reference attached to the US volume in the reference frame of the tracking device. A point, virtually corresponding to the center of the arc of piezo elements is defined and named "US emitter". The proximal neck of the femur is the focus of this study. 10 US volumes were taken: 5 from the anterior and 5 from the posterior femur.

A region growing segmentation, using manual thresholds was first applied on US images. The obtained rough and thick US surface, were then smoothed using Poisson surface reconstruction [8]. We chose to use a ray-casting of the volumes using the spatial location of the US emitter to extract the external surface: points in the volume that are closer to the US emitter. Ray-casting was performed using vtkCellLocator [20]. Segmentation steps are summarized in Fig 2.

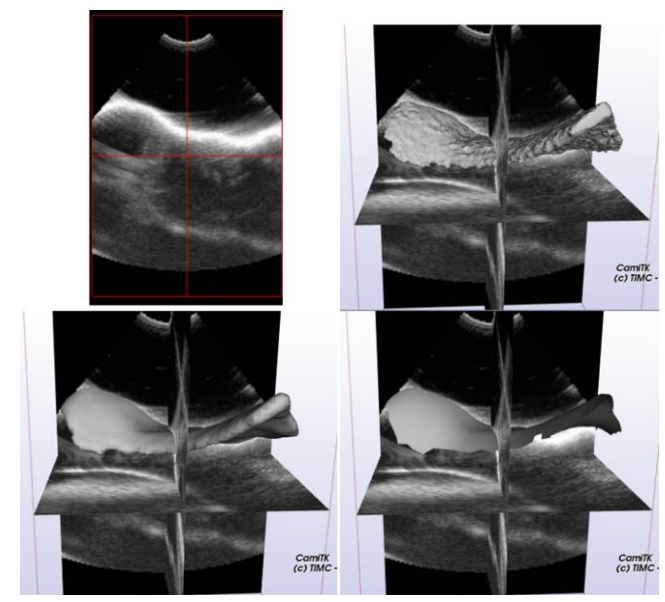

Fig 2: Segmentation steps: A) US image (Top left),

B) Threshold US image (Top right), C) Poisson surface reconstruction (Bottom left), D) Ray-casting (Bottom right).

\subsection{Reference positions}

In order to evaluate the registration results, a ground truth is computed using fiducials attached to the sawbone. Their position is extracted from CT data. Fiducials are represented in the CT volumes as round shapes, and we estimate a fiducial location at the center of mass of its corresponding shape. Then they are digitized using an NDI Spectra Polaris system and transferred to the US reference frame using the US probe calibration information. Fiducials paired-points matching enables bringing the CT scan to the US image in a single reference system (Fig 3 and 4). The mean fiducials matching error is equal to $1.29 \mathrm{~mm}$. The calibration had an average error of $\left(0.50 \mathrm{~mm}, 0.88^{\circ}\right)$. We propose to perform a local systematic research of the minimum US-CT SRE (1), in [+- $\left.2 \mathrm{~mm},+-2^{\circ}\right]$. The final mean (US-CT) SRE at GT is 0.66 $\mathrm{mm}$.

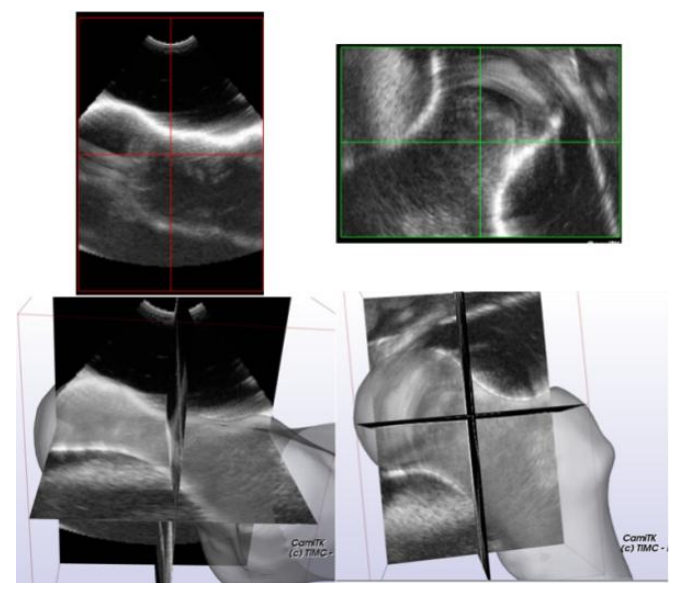

Fig 3: Visualization of the CT surface superimposed on the US volume

\section{Experiments and results}

Initial relative positions of the $\mathrm{CT}$ and US are randomly generated from the ground truth positions. 500 random transformations following normal distribution are generated and applied on the US surfaces in the reference positions. The tested translation parameters are in $[-20 \mathrm{~mm}, 20 \mathrm{~mm}]$ and the rotational angles are in $\left[-90^{\circ}, 90^{\circ}\right]$ (around the 3 principal axes of the US meshes, each transformation parameter is generated independently). Standard ICP and the proposed initialized ICP (I_ICP) are compared.

Target Registration Error (TRE) is defined as the SRE between the registered US mesh and the US mesh on the reference position and this is done knowing the correspondences. The SRE is given following (1) where the set $\mathrm{X}$ is the US mesh and the set $\mathrm{Y}$ is the CT model. Only "successful" registrations (defined as: TRE $<2 \mathrm{~mm}$ ) are considered. Registration is tested on 10 US volumes; 5 are anterior (resp. posterior) and named $\mathrm{Ai}$ (resp. Pi). Quantitative measures of accuracy and runtime are shown in Table 1. With an average of 23 (resp. 15) iterations for A (resp. P), ICP success rates increase from $25 \%$ (resp. 21.1\%) for A (resp. P) to more than $76 \%$ (resp. 52\%) for A (resp.P). ICP accuracy, both with and without initialization, can be clinically accepted. On a 4th generation i5 processor, the registration takes about $3 \mathrm{~s}$, and the mean initialization runtime is nearly twice classical ICP runtime approach. Visualization of random initial states, ICP and I_ICP results are shown in Fig 5. Symmetry descriptors for a 3D shape are provided in [21]. This study does not include such features, but it is clear that the iterative initialization profits from the closed form of the femur. 


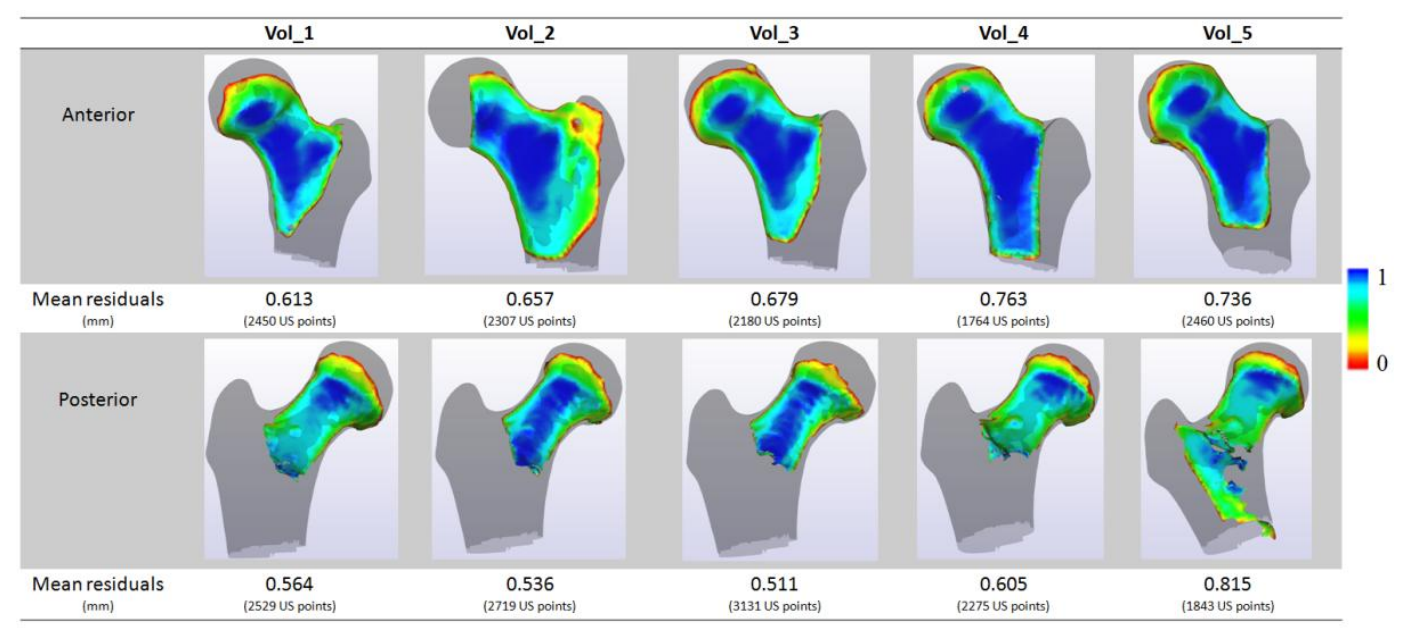

Fig 4: Visualization of the reference positions of the registrations and corresponding mean residuals

\section{Conclusion}

Once calibrated US data are registered to CT, the location of femoral structures in the OR and preoperative plan transfer become possible. Clinical applications of femur recognition in ultrasound volumes are: ultrasound guided femur-tumor ablation, total hip and knee arthroplasty. ICP is a fast and iterative rigid registration method that is sensitive to its initial state. An iterative initialization using combined ICP with annealed filtering is proposed and has been tested for proximal femur recognition in US volumes. Five US acquisitions, by femur side and focusing on the neck of a fixed femur phantom in a water bath, were used in this study along with a CT scan. We show that the use of the full model of the bones as the source helps initializing ICP. Posterior US acquisitions, where the trochanteric crest can hinder the US beam spread, had less successful registrations to CT than anterior acquisitions. Registration runtimes are equivalent to US volume acquisition and construction.
The tested range of capture was significant $[ \pm 20 \mathrm{~mm}$, $\left.\pm 90^{\circ}\right]$, and the obtained success rates make ICP a good candidate for transferring a preoperative plan in CAOS. Among feature based registrations, a sequential least squares estimation technique, the Unscented Kalman Filter (UKF) [22], overpasses ICP on a general case of US bones to CT registration. UKF showed quite worse results than ICP on scapula data [23]. UKF is also computationally demanding compared to ICP. A Genetic algorithm for registering femur data was proposed [24] and it was reported that the obtained results are not impressive as compared to ICP.

More phantoms and US focus zones need to be tested. The effect of the registration error (TRE, SRE) on the intraoperative system feed-back uncertainty is a crucial information for the validation. This requirement depends on the surgery into focus: Bone-tumor localization uncertainty for navigated bone tumor ablation and offset (with the leg length) for navigated total hip arthroplasty.

\begin{tabular}{|c|c|c|c|c|c|c|c|c|c|c|}
\hline \multirow[t]{2}{*}{$\begin{array}{c}\text { US } \\
\text { acquisition }\end{array}$} & \multicolumn{2}{|c|}{$\begin{array}{l}\text { Percentage of successful } \\
\text { registrations (Success criteria: } \\
\text { TRE }<2 \mathrm{~mm} \text { ) }\end{array}$} & \multicolumn{2}{|c|}{$\begin{array}{l}\text { Mean run time } \\
\text { (seconds) }\end{array}$} & \multicolumn{2}{|c|}{$\begin{array}{l}\text { Mean TRE } \\
(\mathrm{mm})\end{array}$} & \multicolumn{2}{|c|}{$\begin{array}{l}\text { Mean SRE } \\
(\mathrm{mm})\end{array}$} & \multicolumn{2}{|c|}{$\begin{array}{l}\text { Mean number of } \\
\text { iterations } \\
\text { (round to nearest) }\end{array}$} \\
\hline & ICP & I-ICP & ICP & I-ICP & ICP & I-ICP & ICP & I-ICP & ICP & I-ICP \\
\hline A1 & 24.6 & 75.8 & 1.16 & 3.15 & 0.37 & 0.21 & 0.612 & 0.610 & 89 & $21+57$ \\
\hline $\mathrm{A} 2$ & 29.8 & 72.8 & 1.56 & 3.88 & 0.21 & 0.17 & 0.641 & 0.640 & 122 & $26+82$ \\
\hline $\mathrm{A} 3$ & 25 & 79.2 & 1.12 & 3.14 & 0.22 & 0.25 & 0.683 & 0.685 & 88 & $22+63$ \\
\hline A4 & 22.4 & 81.6 & 0.79 & 2.59 & 0.37 & 0.23 & 0.768 & 0.767 & 71 & $22+49$ \\
\hline A5 & 23.2 & 75.2 & 1.17 & 3.63 & 0.34 & 0.28 & 0.737 & 0.736 & 87 & $25+58$ \\
\hline Mean (A) & 25 & 76.92 & 1.16 & 3.27 & 0.30 & 0.22 & 0.688 & 0.687 & 91 & $23+62(85)$ \\
\hline P1 & 16.8 & 33.4 & 1.45 & 2.54 & 0.61 & 0.52 & 0.568 & 0.573 & 112 & $12+92$ \\
\hline P2 & 22.4 & 59.2 & 1.70 & 2.80 & 0.59 & 0.60 & 0.541 & 0.540 & 140 & $11+115$ \\
\hline P3 & 21.1 & 62.4 & 1.99 & 3.47 & 0.66 & 0.78 & 0.520 & 0.515 & 155 & $10+158$ \\
\hline P4 & 18.4 & 37 & 1.49 & 2.55 & 0.44 & 0.22 & 0.614 & 0.608 & 127 & $13+98$ \\
\hline P5 & 26.8 & 70.4 & 1.27 & 3.27 & 0.99 & 1.18 & 0.875 & 0.888 & 103 & $27+72$ \\
\hline Mean $(\mathbf{P})$ & 21.1 & 52.48 & 1.58 & 2.92 & 0.65 & 0.66 & 0.623 & 0.624 & 127 & $15+107(122)$ \\
\hline
\end{tabular}

Table 1: Quantitative results 


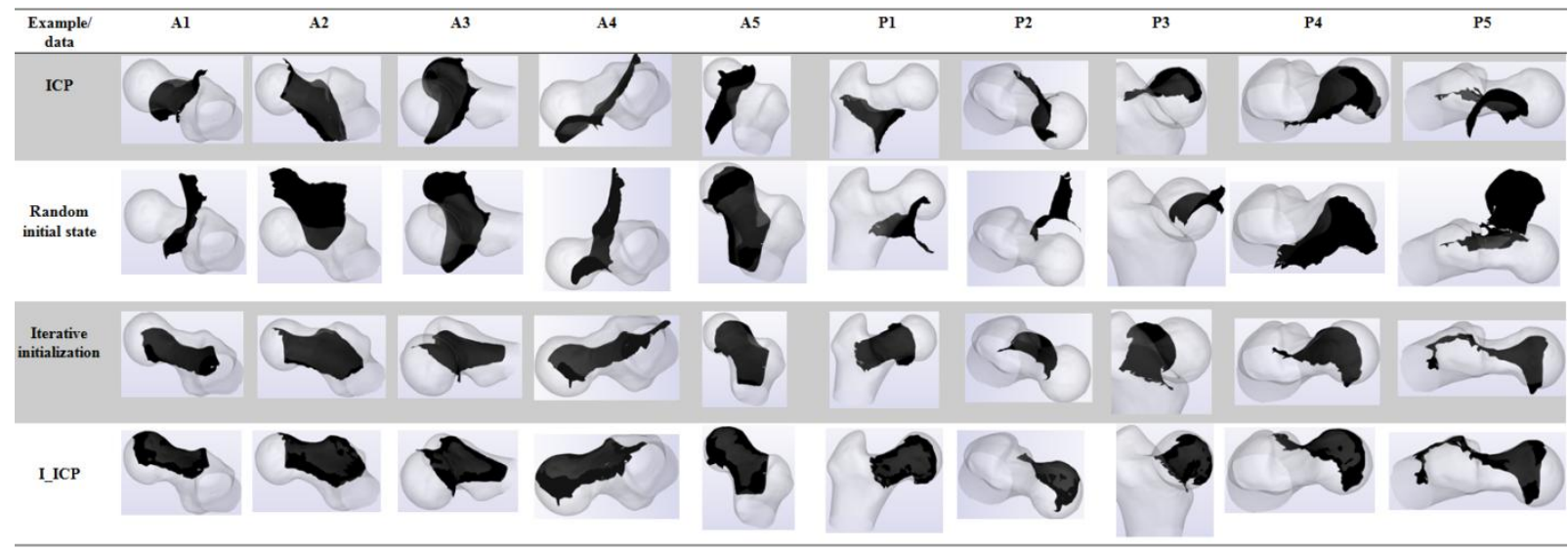

Fig 5: Qualitative results

Acknowledgments: Thanks to Johan Sarrazin, TIMC-IMAG, for providing calibration material and assistance. This work was partly supported by the French ANR within the Investissements d'Avenir program (Labex CAMI) under reference ANR-11-LABX-0004. Computer Assisted Medical Intervention Tool Kit.

\section{References}

[1] A. Mozes. 3D A-Mode Ultrasound Calibration and Registration of the Tibia and Femur for Computer- Assisted Robotic Surgery. PhD thesis, The University of Miami 2008.

[2] P. Besl and N. D. McKay, "A Method for Registration of 3D Shapes." IEEE Transactions on Pattern Analysis and Machine Intelligence, Vol. 14, pp. 239 - 256, 1992.

[3] T. Renkawitz, T. Schuster, J. Grifka, T. Kalteis and E. Sendtner. Leg Length and Offset Measures with a Pinless Femoral Reference Array during THA. Clin Orthop Relat Res, 2009. DOI 10.1007/s11999-009-1086-1

[4] E. Veron-Tocquet, J. Leboucher1, V. Burdin, J. Savéan and O. Rémis-Nérys. A study of accuracy for a single Time of Flight camera capturing knee flexion movement. IEEE Healthcare Innovation Conference (HIC) 2014. Pages: 339 - 342. DOI: 10.1109/HIC.2014.7038944.

[5] J. Schers, C. Plaskos, J. Troccaz, C. Fouard and O. Palombi. 3D/3D Ultrasound registration for panoramic volume reconstruction, Proceedings of CARS'2009 International Journal of ComputerAided Radiology and Surgery, vol.4, Supp.1, june 2009, p100-101.

[6] M. Betke, H. Hong and J. P. Ko. Automatic 3D Registration of Lung Surfaces in Computed Tomography Scans, Medical Image Computing and Computer Assisted Intervention (MICCAI), 2001. LNCS 2208, pp. 725-733, 2001.

[7] P. Krsek, T. Pajdla, and V. Halvac. Differential invariants as the base of triangulated surface registration. Computer Vision and Image Understanding 87(1-3), 27 - 38 (2002).

[8] H. E. Fakhfakh, G. Llort-Pujol, C. Hamitouche and E. Stindel. Automatic registration of pre- and intraoperative data for long bones in Minimally Invasive Surgery. 36th Annual International Conference of the IEEE EMBC 2014, Page(s):5575 - 5578.
[9] O. Haddad. J. Leboucher, J. Troccaz and E. Stindel. Robust rigid registration for non invasive Computer Assisted Orthopedic Surgery. Preliminary results. Biomedical Imaging (ISBI), 2015 IEEE 12th International Symposium on, Page(s): 725 - 729.

[10] E. Lomonosov, D. Chetverikov and A. Ekárt. Pre-registration of arbitrarily oriented 3D surfaces using a genetic algorithm. Pattern Recognition Letters, volume 27, Issue 11, August 2006, Pages 1201-1208.

[11] T. N. Linha and H. Hiroshib. Global iterative closet point using nested annealing for initialization. Knowledge Based and Intelligent Information and Engineering Systems, Procedia Computer Science 60 (2015) $381-39$.

[12] L. Fieten, K. Radermacher and S. Heger. An ICP variant with anisotropic weighting to accommodate measurement errors in A-Mode ultrasound-based registration. Biomed Tech 2012.

[13] X. Ye, J. A. Noble and D. Atkinson. 3-D Freehand Echocardiography for Automatic Left Ventricle Reconstruction and Analysis Based on Multiple Acoustic Windows. IEEE TRANSACTIONS ON MEDICAL IMAGING, VOL. 21, NO. 9, SEPTEMBER 20021051

[14] M. Baumann, V. Daanen, A. Leroy and J. Troccaz. 3-D Ultrasound Probe Calibration for Computer-Guided Diagnosis and Therapy. CVAMIA 2006, LNCS 4241, pp. 248-259, 2006.

[15] A. Clavé, F. Fazilleau, D. Cheval, T. Williams, C. Lefèvre and E. Stindel. Comparison of the reliability of leg length and offset data generated by three hip replacement CAOS systems using EOS ${ }^{\mathrm{TM}}$ imaging. Orthop Traumatol Surg Res. 2015 Oct;101(6):64753.

[16] S. Billings and R. Taylor. Iterative Most Likely Oriented Point Registration. MICCAI 2014, Part I, LNCS 8673, pp. 178-185, 2014.

[17] I. Hacihaliloglu, R. Abugharbieh, A. Hodgson, and R. Rohling. Bone Segmentation and Fracture Detection in Ultrasound Using 3D Local Phase Features. MICCAI 2008, Part I, LNCS 5241, pp. $287-$ 295, 2008.

[18] B. Bellekens, V. Spruyt, R. Berkvens and Maarten Weyn. A Survey of Rigid 3D Pointcloud Registration Algorithms. AMBIENT 
2014: The Fourth International Conference on Ambient Computing, Applications, Services and Technologies. ISBN: 978-1-61208-3568.

[19] P. Cignoni, M. Callieri, M. Corsini, M. Dellepiane, F. Ganovelli and G. Ranzuglia. MeshLab: an Open-Source Mesh Processing Tool P. Eurographics Conference (2008).

[20] W. J. Schroeder, L. S. Avila and W. Hoffman. Visualizing with VTK: A Tutorial. IEEE Computer Graphics and Applications 2000.

[21] M. Kazhdan, T. Funkhouser and S. Rusinkiewicz. Symmetry Descriptors and 3D Shape Matching. Eurographics Symposium on Geometry Processing (2004)
[22] M. Hedjazi Moghari and P. Abolmaesumi. A Novel Incremental Technique for Ultrasound to CT Bone Surface Registration Using Unscented Kalman Filtering. MICCAI 2005.

[23] K. Tyryshkin, P. Mousavi, DR. Pichora and P. Abolmaesumi. Identification of anatomical landmarks for registration of CT and ultrasound images in computer-assisted shoulder arthroscopy. IEEE EMBS 2006.

[24] R. Uddanwadikar, V. Uddanwadiker and S. Chiddarwar. Fabrication of Accurate Bone Implant Geometry Using Puzzle Solving Technqiue. Proceedings of the 1st International Conference on Machines and Mechanisms 2013. 